\title{
Asymmetry of SPECT Perfusion Image Patterns as a Diagnostic Feature for Alzheimer's Disease
}

\author{
Vassili A. Kovalev ${ }^{1}$, Lennart Thurfjell ${ }^{2}$, Roger Lundqvist ${ }^{2}$, and Marco Pagani ${ }^{3}$ \\ ${ }^{1}$ Centre for Vision, Speech and Signal Processing \\ University of Surrey, Guildford, Surrey GU2 7XH, United Kingdom \\ ${ }^{2}$ Centre for Image Analysis, Uppsala University, SE-752 37 Uppsala, Sweden \\ ${ }^{3}$ Institute of Cognitive Sciences and Technologies, CNR, 00185 Rome, Italy
}

\begin{abstract}
In this paper we propose a new diagnostic feature for Alzheimer's Disease (AD) which is based on assessment of the degree of inter-hemispheric asymmetry using Single Photon Emission Computed Tomography (SPECT). The asymmetry measure used represents differences in 3D perfusion image patterns in the cerebral hemispheres. We start from the simplest descriptors of brain perfusion such as the mean intensity within pairs of brain lobes, gradually increasing the resolution up to five-dimensional co-occurrence matrices. Evaluation of the method was performed using SPECT scans of 79 subjects including 42 patients with clinical diagnosis of $\mathrm{AD}$ and 37 controls. It was found that combination of intensity and gradient features in co-occurrence matrices captures significant differences in asymmetry values between AD and normal controls $(p<0.00003$ for all cerebral lobes). Our results suggest that the asymmetry feature is useful for discriminating $\mathrm{AD}$ patients from normal controls as detected by SPECT.
\end{abstract}

\section{Introduction}

PET and SPECT have been widely used to investigate alterations of functional patterns in patients with Alzheimer's Disease (AD). A decrease in regional cerebral blood flow $(\mathrm{rCBF})$ in the parieto-temporal cortex is considered to be a diagnostic sign of AD. Nevertheless, in the early stage of the disease the typical bilateral parieto-temporal $\mathrm{rCBF}$ deficit is seldom described and there is a certain degree of hypo-perfusion distribution overlap between $\mathrm{AD}$ patients and normal elderly. In the past, the diagnosis of $\mathrm{AD}$ was often based on visual examination of the images or on other subjective approaches such as analysis of manually placed regions of interests (ROIs) [1. Since the introduction of semi-quantitative and 3D standardisation methods in SPECT image analysis visual reading has been less and less utilised by all research groups. Objective methods reported in literature include methods based on pixel-by-pixel comparison of a patient's scan to data from normal volunteers [2], 3], the analysis of ROIs or VOIs (volumes of interest) defined by a stereotactic atlas registered to the data (eg, 4), and the most advanced SPM method 5 . These methods require a preceding spatial registration of the data (eg, [3], 6]). Moreover, the data must be normalized for 
global activity. Different approaches have been used and for most methods the main assumption is to normalize the data against the activity in areas that are unaffected by the disease (see, for example, [7]).

In order to overcome these difficulties we propose to use a measure that capitalise on the fact that, although a bilateral flow reduction in the parieto-temporal cortex is an important diagnostic feature for AD, there is normally an asymmetry in the degree of involvement 3. AD often starts in one hemisphere and gradually becomes more bilateral during the progression of the disease. Because of this we hypothesize that if we consider a healthy person that becomes affected by $\mathrm{AD}$, one would expect a low asymmetry to start with. Then the asymmetry index would gradually increase during the early phase when the disease mainly involves one hemisphere [8, 9, 10, and finally that the asymmetry index would decrease when there is a bilateral involvement. Hence, an asymmetry index that captures specific differences in spatial structure of bilateral three-dimensional (3D) perfusion patterns could be considered as a diagnostic feature of AD.

Previous work on perfusion asymmetry is mainly restricted to the computation of ratios of VOIs where an asymmetry index is computed as |right left $\mid /($ right + left $)$, where right and left indicate values within VOIs placed in both hemispheres 4. More recently, a new kind of multi-sort co-occurrence matrices has been suggested to properly describe the three-dimensional spatial structure (texture) of medical images [1]. These descriptors were first used for the analysis of MRI datasets [12, 13, and later on for direct inter-subject comparisons of SPECT scans [14.

In this paper, we systematically investigate and compare the usefulness of new asymmetry features derived from SPECT perfusion scans. We start from the simplest and most obvious characteristics of brain perfusion such as the mean intensity within different anatomical regions, gradually increasing the resolution through vector (histogram) features, and going up to five-dimensional intensity/gradient co-occurrence matrices. All co-occurrence matrices used are independent of translation, rotation, reflection, and size of the 3D regions they describe 11. The analysis was performed implementing the Greitz Computerized Brain Atlas 15 to standardize the scans, define the regions of interest (cerebral lobes), and normalize the image data. To our best knowledge, this is the first work on examining the diagnostic abilities of asymmetry indices obtained by means of comparison of $3 \mathrm{D}$ perfusion image patterns using co-occurrence matrices.

\section{Method}

\subsection{Subjects and SPECT Scans}

Alzheimer's patients were selected from all consecutive ones referred to the Department of Nuclear Medicine of Karolinska Hospital, Stockholm for suspected AD. The AD group consisted of 42 patients (22 males and 20 females, mean age $74 \pm 7$ years) with cognitive decline suggestive of AD. The clinical diagnosis of AD was clinically determined, fulfilling the NINCDS-ADRDA criteria 

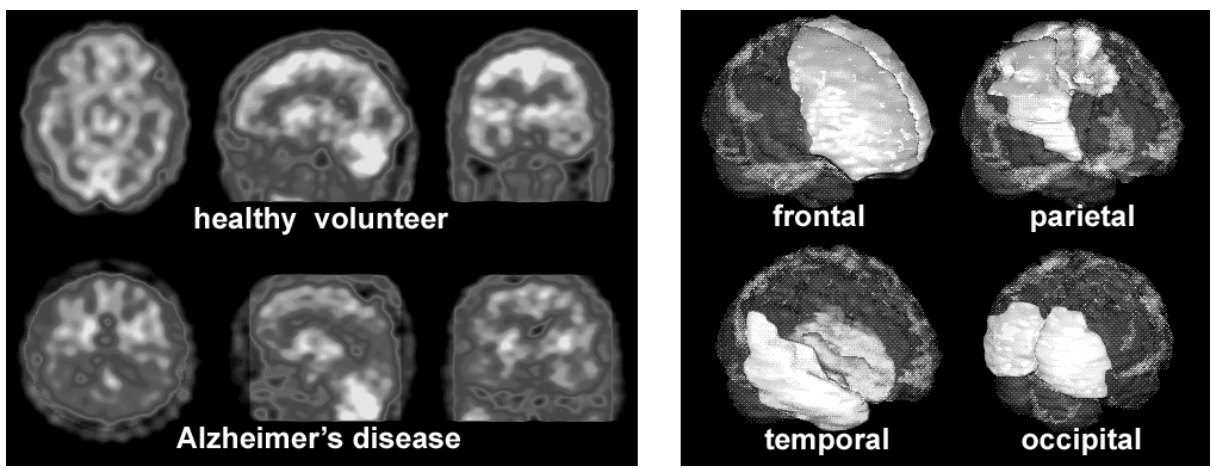

Fig. 1. Left panel: examples of original SPECT scans (axial, sagittal, and coronal sections are shown from left to right). Right panel: pairs of brain lobes.

for possible AD, with a MMSE score less than 20 and being also supported by Computer Tomography (CT) and Electroencephalographic (EEG) changes. The rCBF values of these patients were compared to 37 normal controls (18 males, 19 females, mean age $63 \pm 12$ years) chosen from the library of normal scans available at the Department in order to match as much as possible age and gender of AD patients. Patients and normal volunteers were scanned under the same physiological conditions at rest and with the eyes closed. The slight difference in mean age due to the difficulty in recruiting too old control subjects did not significantly affect the $\mathrm{rCBF}$ in the VOIs under study. About $1000 \mathrm{MBq} 99 \mathrm{~m}$ Tc-HMPAO were prepared and was administered intravenously to the subjects within 5 minutes from the preparation. SPECT brain imaging was performed using a three-headed Gamma Camera (TRIAD XLT 20, Trionix Research Laboratory Inc., Twinsburg, OH, USA) equipped with a low-energy high-resolution (LEHR) collimator. The projection data were acquired for 30 seconds per projection at 90 equal angles of a complete revolution. The SPECT images were reconstructed by filtered back projection algorithm using a ramp filter with a cut-off frequency of 0.6 cycles/cm. The data were smoothed using a Hamming filter with a cut-off frequency of 2.25 . The attenuation correction was based on a four-point ellipse. Data were projected into a 128x128 pixel matrix that resulted in an isotropic voxel size of $2.2 \mathrm{~mm}$. Examples of original scans are shown in the left panel of Fig 1 .

\subsection{Brain Regions and Asymmetry Evaluation Procedure}

The asymmetry features were obtained by comparing certain measures within pairs of anatomical regions. For definition of these anatomical regions (VOIs), the Greitz Computerized Brain Atlas [15] was used. For registration of the atlas to each of the individual SPECT scans, a fully automated method was used to register a SPECT template volume previously reformatted to atlas space with the individual SPECT scan. Details of the registration procedure can be found 
in [14]. The brain regions considered in this work were the frontal, parietal, temporal, occipital and cerebellar lobes (Fig 10). Similar to previous asymmetry study in normal population using MRI [12, the asymmetry was regarded here as dissimilarity of 3D perfusion patterns, as detected by SPECT, within pairs of lobes in the left and right brain hemispheres. It should be stressed that there are no direct relationships between the asymmetry of 3D volumetric image patterns and the shape of 3D surfaces they are bounded with.

For all kinds of features under consideration, we are measuring the degree of dissimilarity as the Euclidean distance between these features. Comparison of image features computed for pairs of brain lobes resulted in a single dissimilarity value treated as an asymmetry index. The primary goal was to evaluate the usefulness of different image features (descriptors) and relative importance of different anatomical regions for discrimination of $\mathrm{AD}$ and controls. We start from the simplest and most obvious characteristics of brain perfusion such as the mean intensity within different anatomical regions, gradually increasing the resolution through vector (histogram) features, and going up to five-dimensional intensity/gradient co-occurrence matrices. The list of features examined in this study includes: (a) the mean intensity and 3D gradient [16] values, (b) the intensity and gradient magnitude histograms (16 bins each), (c) the three-dimensional intensity co-occurrence matrices $W 1$, (d) the three-dimensional gradient cooccurrence matrices $W 2$, (e) the combined, five-dimensional intensity and gradient co-occurence matrices $W 3$ that provide detailed description of spatial structure of $3 \mathrm{D}$ perfusion patters in brain lobes.

The combined $W 3$ matrix of an image region is a five-dimensional array [14]. The matrix axes are associated with elementary features of voxel pairs. All possible neighbors around each voxel with no repetition were considered for given distance range so that descriptors are reflection- and rotation-invariant. More formally, let us consider an arbitrary voxel pair $(i, k)$ defined in 3D by voxel indices $i=\left(x_{i}, y_{i}, z_{i}\right)$ and $k=\left(x_{k}, y_{k}, z_{k}\right)$ and with inter-voxel distance $d(i, k)$. Let us denote their intensities by $I(i)$ and $I(k)$, local gradient magnitudes by $G(i), G(k)$. Then the $W 3$ co-occurrence matrix can be defined as:

$$
W 3=\|w(I(i), I(k), G(i), G(k), d(i, k))\|,
$$

where $w$ denotes the number of voxel pairs with characteristics given in brackets. The intensity gradients at each voxel positions were computed using a 3D filter with the small $3 \times 3 \times 3$ window suggested in [16]. Further implementation details can be found in 11. The three-dimensional matrices $W 1$ and $W 2$ are defined in a similar way and may be viewed as particular cases of the more general $W 3$ matrix. The intensity and gradient values were quantized down to 16 bins and inter-voxel distance varied within 1-5 bins, $2.2 \mathrm{~mm}$ each.

The feature evaluation procedure includes the following three steps:

(a) fractional ranking of the tested asymmetry measure values across all SPECT scans to the range of 0-100 to allow direct inter-region comparison,

(b) evaluation of the significance of the asymmetry features using $t$-test and K-means clustering, 
(c) visualization and analysis of subjects' scattering in asymmetry feature space using reduced, two-dimensional scatter plots obtained with the help of multidimensional scaling method [17.

These steps were performed for all types of features given above. The $t-$ and $p$-values were used as quantitative estimates of the ability of a tested feature to capture the asymmetry property in the image. In all the analyses the cooccurrence matrices treated as corresponding feature vectors.

\section{Results}

Our results suggest that the mean intensity within the brain lobes did not provide any useful information for discriminating $\mathrm{AD}$ and normal controls based on their asymmetry. As a result of two-tailed $t$-test, no pair of lobes showed any statistical significance of the hypothesis. $T$-values were unacceptably small (in order of 0.3 ) with very high $p$-levels ranged from 0.41 for occipital lobes to as high as 0.90 for frontal lobes. Asymmetry values computed using mean gradient magnitudes gave slightly better results but $p$-levels were still unacceptably high (from 0.06 for occipital lobes to 0.73 for temporal lobes). The $t$-test performed on intensity and gradient histogram features implied that the best region was the occipital lobe ( $p$-values of 0.11 for intensity and 0.04 for gradient). However, no region demonstrated any significant difference in asymmetry between AD and controls with these features.

Results summarized in Table 1 evidencing that the hypothesis of the existence of different asymmetry for $\mathrm{AD}$ patients and controls cannot be rejected. This is the case for asymmetry computed using both kind of matrices $W 1$ and $W 2$. A comparison of $t$-values and $p$-levels suggests that the most important anatomical regions for measuring asymmetry were the occipital and the temporal lobes while the cerebellum did not provide any useful information at all. Furthermore, results obtained using combined 5D intensity/gradient matrices (see Table 1) confirmed the existence of a significant difference in asymmetry values for AD as compared to normal controls for all lobes except for the cerebellum. The data reported in two last columns of the table suggest that the most useful regions for discriminating $\mathrm{AD}$ from normal controls are the temporal lobes. This is

Table 1. Significance of asymmetry differences between AD patients and controls

\begin{tabular}{|c|c|c|c|c|c|c|}
\hline \multirow{2}{*}{$\begin{array}{c}\text { brain } \\
\text { lobe } \\
\text { pair }\end{array}$} & \multicolumn{2}{|c|}{$\begin{array}{c}\text { intensity co-occurrence } \\
\text { matrices } W 1\end{array}$} & \multicolumn{2}{|c|}{$\begin{array}{r}\text { gradient co-occurrence } \\
\text { matrices } W 2\end{array}$} & \multicolumn{2}{c|}{$\begin{array}{c}\text { combined co-occurrence } \\
\text { matrices } W 3\end{array}$} \\
\cline { 2 - 7 } & $t$-value & $p$-value & $t$-value & $p$-value & $t$-value & $p$-value \\
\hline \hline Frontal & 2.15 & 0.034 & 4.29 & $<0.001$ & 5.15 & $<0.00003$ \\
Parietal & 3.50 & 0.001 & 5.72 & $<0.001$ & 6.20 & $<0.00001$ \\
Occipital & 6.36 & $<0.001$ & 7.05 & $<0.001$ & 8.51 & $<0.00001$ \\
Temporal & 5.21 & $<0.001$ & 7.00 & $<0.001$ & 9.36 & $<0.00001$ \\
Cerebellum & 1.10 & 0.274 & 0.86 & 0.090 & 0.92 & 0.33 \\
\hline
\end{tabular}


Table 2. Mean and STD values of lobular asymmetry (combined matrices $W 3$ )

\begin{tabular}{|c|c|c|c|c|}
\hline \multirow{2}{*}{$\begin{array}{c}\text { brain } \\
\text { lobe } \\
\text { pair }\end{array}$} & \multicolumn{4}{|c|}{ asymmetry value } \\
\cline { 2 - 5 } & normal controls & \multicolumn{2}{|c|}{ AD patients } \\
\cline { 2 - 5 } & mean & STD & mean & STD \\
\hline \hline Frontal & 35.0 & 25.0 & 64.4 & 25.4 \\
Parietal & 32.9 & 22.7 & 66.3 & 24.9 \\
Occipital & 29.2 & 20.4 & 69.5 & 21.5 \\
Temporal & 28.2 & 18.8 & 70.4 & 20.0 \\
\hline
\end{tabular}

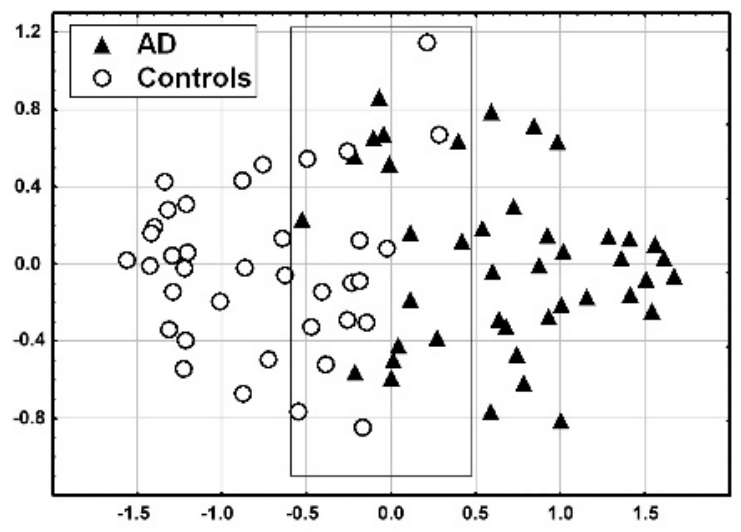

Fig. 2. Scatter plot of Alzheimer's cases and normal controls in asymmetry feature space. The original four-dimensional space constituted by asymmetry indices computed for four pairs of brain lobes is reduced to two conditional dimensions using multidimensional scaling method. The "neutral zone" where AD patients and controls are mixed is bounded by the rectangle.

further illustrated by mean and STD values summarized in Table 2 (the cerebellum is omitted as insignificant). The asymmetry values computed for frontal, parietal, temporal, and occipital lobe pairs of each subject form a four-dimensional feature space. A scatter plot of the asymmetry of all AD cases and controls obtained by reducing the four-dimensional feature space down to two dimensions using multidimensional scaling method is shown in Fig. 2, Interestingly, when an unsupervised K-means clustering method is applied to the data to cluster them down for three groups, the following classes were obtained: class 1 (27 cases, AD patients only), class 2 (21 cases, normal controls only), class 3 (31 cases, $15 \mathrm{AD}$ and 16 control cases mixed). These results are well in agreement with the data presented in Fig. 2. Thus, with this unbiased classification strategy, 48 cases $(60.8 \%)$ were categorized to the right classes and the remaining 31 cases were categorized to some sort of "uncertain" class but not missclassified. 


\section{Discussion}

Our result suggest that the mean and histogram features of intensity and gradient magnitude computed for brain lobes do not provide enough resolution to describe brain activity asymmetry usable for discrimination of AD and control scans. This is not surprising because all these features do not utilize spatial information constituted by the specific intensity distribution within brain lobes. Nevertheless, the simplest of these features, namely the mean intensity within predefined regions, is the most commonly used feature in previous work on AD asymmetry in this context 1, 4. The choice of 3D large VOIs (entire lobes in our case) instead of 2D ROIs renders the analysis more sensitive to changes in perfusion (and hence to asymmetry) and less dependent on errors in outlining and positioning. The results obtained using specific co-occurrence matrices for intensity and gradient values within the different brain lobes gave much better group separation ( $p$-values ranged from 0.034 up to 0.001 ). The main reason for improvements is perhaps that matrices take also the spatial information into account. When comparing intensity and gradient information, gradient information tends to be more important for asymmetry estimation. This trend was observed across all the tests performed on individual features. From our results, it seems that the rate of change in intensity is more useful than the intensity level itself for discrimination between $\mathrm{AD}$ and controls. The best results were obtained when intensity and gradient features were combined into 5D co-occurrence matrices. The differences in asymmetry values between patients and controls found to be highly significant $(p<0.00003$ for all four cerebral lobes). Thus, our results support the research hypothesis about the importance of 3D asymmetry index as a diagnostic feature of AD. Similar trend of asymmetry increase in AD was also found using high resolution anatomical MRI datasets [11. The decrease of asymmetry at the late stages with bilateral involvement needs to be studied separately using an additional SPECT material.

In conclusion, we believe that the high-resolution asymmetry measures we propose, provide very useful information for discrimination of AD and normal controls. It may not be the ultimate feature but is likely to be more objective and robust than previous approaches. Furthermore, the asymmetry measure is independent of the intensity scale of the SPECT datasets and all inter-subject comparisons are made on the level of asymmetry features only. Therefore there should be no need for normalization of global activity. However, further blind studies are needed to clinically validate the new methodological approach proposed in this paper. Finally, we would like to emphasize that any asymmetry measure is not alone sufficient as a diagnostic feature for discrimination of $\mathrm{AD}$ from controls. Instead it gives additional information and should be used in conjunction with the more traditional methods for discriminating $\mathrm{AD}$ from controls e.g. through the comparison with a normals database. 


\section{References}

1. Ishii, K., Sasaki, M., Yamaji, S., Sakamoto, S., Kitagaki, H., Mori, E.: Paradoxal hippocampus perfusion in mild-to-moderate alzheimer's disease. J Nucl Med 39 (1998) 293-298

2. Bartenstein, P., Minoshima, S., Hirsch, C., Buch, K., Willoch, F., Mosch, D., Schad, D., Schwaiger, M., Kurz, A.: Quantitative assessment of cerebral blood flow in patients with Alzheimer's disease by SPECT. J Nucl Med 38 (1997) 1095-1101

3. Minoshima, S., Frey, K., Koeppe, R., Foster, N., Kuhl, D.: A diagnostic approach in Alzheimer's disease using three-dimensional stereotactic surface projections of fluorine-18-FDG PET. J Nucl Med 36 (1995) 1238-1248

4. Borght, T., Minoshima, S., Giordani, B., Foster, N., Frey, K., Berent, S., Albin, R., Koeppe, R., Kuhl, D.: Cerebral metabolic differences in Parkinson's and Alzheimer's disease matched for dementia severity. J Nucl Med 38 (1997) 797-801

5. Ashburner, J., Friston, K.J.: Nonlinear spatial normalization using basis functions. Hum Brain Mapp 7(4) (1999) 254-266

6. Andersson, J., Thurfjell, L.: Implementation and validation of a fully automatic system for intra- and inter-individual registration of PET brain scans. J Comput Assist Tomogr 21(1) (1997) 136-144

7. Andersson, J.L.: How to estimate global activity independent of changes in local activity. NeuroImage 6(4) (1997) 237-244

8. Celsis, P., Agniel, A., Cardebat, D., Demonet, J.F., Ousset, P.J., Puel, A.: Age related cognitive decline: a clinical entity? A longitudinal study of cerebral blood flow and memory performance. J Neurol Neurosurg Psych 62 (1997) 601-608

9. Talbot, P.R., Snowden, J.S., Lloyd, J.J., Neary, D., Testa, H.J.: The contribution of single photon emission tomography to the clinical differentiation of degenerative cortical brain disorders. J Neurol 242(9) (1995) 579-586

10. Small, G.W., Mazziotta, J.C., Collins, M.T., Baxter, L.R., Phelps, M.E., Mandelkern, M.A., Kaplan, A., Rue, A.L., Adamson, C.F., Chang, L.: Apolipoprotein E type 4 allele and cerebral glucose metabolism in relatives at risk for familial Alzheimer disease. JAMA 273(12) (1995) 942-947

11. Kovalev, V.A., Kruggel, F., Gertz, H.J., von Cramon, D.Y.: Three-dimensional texture analysis of MRI brain datasets. IEEE TMI 20(5) (2001) 424-433

12. Kovalev, V.A., Kruggel, F., von Cramon, D.Y.: Gender and age effects in structural brain asymmetry as measured by MRI texture analysis. NeuroImage 19 (2003) 895-905

13. Kovalev, V.A., Petrou, M., Suckling, J.: Detection of structural differences between the brains of schizophrenic patients and controls. Psych Res 124 (2003) 177-189

14. Pagani, M., Kovalev, V.A., Lundqvist, R., Jacobsson, H., Larsson, S.A., Thurfjell, L.: A new approach for improving diagnostic accuracy of Alzheimer's disease and Frontal Lobe Dementia utilising the intrinsic properties of the SPET datasets. Eur J Nuc Med Molec Imag 30(11) (2003) 1481-1488

15. Greitz, T., Bohm, C., Holte, S., Eriksson, L.: A computerized brain atlas: construction, anatomical content and some applications. J Comp Assist Tomogr 15 (1991) 26-38

16. Zucker, S.W., Hummel, R.A.: A 3D edge operator. IEEE PAMI 3 (1981) 324-331

17. Cox, T.F., Cox, M.A.A.: Multidimensional scaling. 2nd edn. Volume 88 of Monographs on Statistics and Applied Probability. Chapman and Hall, Boca Raton, Florida (2000) 328 p. 